\title{
Diagnostic value of FDG PET/CT imaging in patients with surgically managed infective endocarditis: results of a retrospective analysis at a tertiary center
}

\author{
Sabine Julia Maria Sag, MD, ${ }^{\mathrm{a}}$ Karin Menhart, $M D,{ }^{\mathrm{b}}$ Jirka Grosse, $M D{ }^{\mathrm{b}}$ \\ Florian Hitzenbichler, $M D,{ }^{c}$ Frank Hanses, $M D,{ }^{c}$ Arno Mohr, $M D,{ }^{c}$ \\ Bernd Salzberger, $M D,{ }^{c}$ Matthäus Zerdzitzki, $M D,{ }^{d}$ Michael Hilker, $M D{ }^{d}$ \\ Leopold Rupprecht, $M D,{ }^{d}$ Dirk Hellwig, MD, ${ }^{\mathrm{b}}$ Christof Schmid, $M D,{ }^{d}$ \\ Lars Siegfried Maier, $M D,{ }^{a}$ and Can Martin Sag, $M^{a}$ \\ a Department of Internal Medicine II, University Hospital Regensburg, Regensburg, Germany \\ b Department of Nuclear Medicine, University Hospital Regensburg, Regensburg, Germany \\ c Department of Infection Prevention and Infectious Diseases, University Hospital Regensburg, \\ Regensburg, Germany \\ d Department of Cardiothoracic Surgery, University Hospital Regensburg, Regensburg, Germany
}

Received Jun 17, 2020; accepted Nov 16, 2020

doi: $10.1007 / \mathrm{s} 12350-020-02457-x$

Background. We assessed the diagnostic value of FDG PET/CT in a real-world cohort of patients with surgically managed infective endocarditis (IE).

Methods. We performed a retrospective analysis of all patients hospitalized in a tertiary IE referral medical center from January 2014 to October 2018 fulfilling the following criteria: ICD-10 code for IE and OPS code for both, heart surgery and FDG PET/CT.

Results. Final analysis included 29 patients, whereof 28 patients had surgically proven IE. FDG PET/CT scan was true-positive in 15 patients (sensitivity (SEN) $56 \%$ ) and false-negative in 12 patients. Combination of Duke criteria (DC) with FDG PET/CT scan resulted in gain of SEN for all patients with confirmed IE (SEN of DC 79\% vs SEN of combination DC and FDG PET/ CT 89\%), driven by a relevant gain in PVE patients only (SEN of DC $78 \%$ vs SEN of combination DC and FDG PET/CT 94\%). Interestingly, higher prosthesis age was observed in patients with false-negative scans.

Conclusions. We found a SEN of $56 \%$ for FDG PET/CT in a real-world cohort of patients with surgically proven IE which was associated with a $16 \%$ gain of IE diagnosis in patients with PVE when combined with DC. (J Nucl Cardiol 2022;29:1191-204.)

Key Words: Inflammation • PET • image interpretation

The authors of this article have provided a PowerPoint file, available for download at SpringerLink, which summarizes the contents of the paper and is free for reuse at meetings and presentations. Search for the article DOI on SpringerLink.com.

The authors have also provided an audio summary of the article, which is available to download as ESM, or to listen to via the JNC/ASNC Podcast.

Supplementary Information The online version of this article (h ttps://doi.org/10.1007/s12350-020-02457-x) contains supplementary material, which is available to authorized users.
Reprint requests: Can Martin Sag, MD, Department of Internal Medicine II, University Hospital Regensburg, Franz-Josef-Strauß-Allee 11, 93053 Regensburg, Germany; can-martin.sag@ukr.de $1071-3581 / \$ 34.00$

Copyright (C) 2020 The Author(s) 


\begin{tabular}{|ll|}
\hline Abbreviations \\
CDRIE & $\begin{array}{l}\text { Cardiac device-related infective } \\
\text { endocarditis }\end{array}$ \\
DC & Duke criteria \\
ESC & European Society of Cardiology \\
FDG & ${ }^{18}$ F-fluorodeoxyglucose \\
ICD-10 & International Classification of Diseases \\
IE & Infective endocarditis \\
NVE & Native valve endocarditis \\
PVE & Prosthetic valve endocarditis \\
SEN & Sensitivity \\
SUVmax & Maximal standardized uptake value \\
\hline
\end{tabular}

\section{INTRODUCTION}

Despite medical advancements management of infective endocarditis (IE) is still challenging both from a diagnostic as well as from a therapeutic point of view. Particularly, diagnostic work up in case of suspected prosthesis valve endocarditis (PVE) can be errorprone. $^{1,2}$ Therefore, 2015 ESC IE guidelines incorporated further imaging modalities, including cardiac FDG PET/CT for detection of abnormal periprosthetic inflammation activity, to improve diagnostic accuracy of Duke criteria (DC). ${ }^{3}$ Notably, ESC guidelines are based on studies in which IE diagnosis in PVE and cardiac device-related infective endocarditis (CDRIE) patients was predominantly made by expert teams, respectively, by Duke classification. ${ }^{4-6}$ Likewise, more recent studies investigating the diagnostic value of FDG PET/CT in suspected IE have mainly used expert opinion or DC as reference standard for definite IE diagnosis. ${ }^{7-10}$ However, an assessment of the diagnostic value of FDG PET/ CT via surgical confirmation as reference standard for definite IE is largely missing. This lack of definite surgical diagnosis may complicate interpretation of FDG PET/CT sensitivity (SEN) and specificity, which could have contributed to the high variation of FDG PET/CT SEN in the context of IE diagnostics. ${ }^{4-10}$

The aim of this retrospective analysis was to evaluate the diagnostic value of preoperatively performed PET imaging during a 4 years observation period in a real-world cohort of patients undergoing heart surgery due to suspected IE at a tertiary IE referral center. Furthermore, we wanted to investigate the potential gain of SEN by including PET results into traditional Duke classification for identifying IE patients. In addition, we aimed to investigate confounders that resulted in false-negative or false-positive FDG PET/CT results.

\section{METHODS}

\section{Patient cohort}

We retrospectively reviewed medical records of all patients hospitalized in a tertiary IE referral medical center from January 2014 to October 2018 fulfilling the following criteria: International Classification of Diseases (ICD)-10 code for IE and code for both, heart surgery and FDG PET/CT according to the German classification of operations and procedures (OPS code). Only patients with preoperatively performed FDG PET/ $\mathrm{CT}$ and surgically proven diagnosis were included. The institutional Ethics committee approved this study and waived the necessity to obtain informed consent.

Demographic, microbiological and echocardiographic data of all included patients were gathered.

\section{FDG PET/CT imaging and image interpretation}

In 17 patients a Biograph 16 PET/CT scanner (CTISiemens, Erlangen, Germany) consisting of a 16-slice multidetector CT (.5 s per revolution) was used; in 12 patients a Biograph mCT 40 FLOW PET/CT scanner (CTI-Siemens, Erlangen, Germany), consisting of a 40slice multidetector CT (.5 s per revolution) was used.

After a fasting period of at least 6 hours, $3 \mathrm{MBq}$ ${ }^{18}$ F-FDG per kilogram body weight were injected intravenously $(254 \pm 43 \mathrm{MBq})$. Please note that no specific dietary requirements such as a low-carb/ highfat diet were recommended to patients. The patients' blood glucose level was strictly controlled to be below $150 \mathrm{mg} / \mathrm{dL}(8.32 \mathrm{mmol} / \mathrm{L})$. To increase renal tracer elimination, patients received an injection of $20 \mathrm{mg}$ furosemide as well as intravenous hydration shortly after ${ }^{18}$ F-FDG injection.

To minimize muscular ${ }^{18}$ F-FDG uptake, patients were advised to stay in a quiet lying position. Warming blankets were used to avoid freezing of the patients and to keep potential tracer accumulation in brown fat tissue to a minimum. Patients were instructed to void the bladder prior to scanning and to remove all metal parts.

After a waiting period of about 60 minute postinjection, the PET/CT acquisition was performed. Using the Biograph 16 PET/CT scanner, images of the trunk were acquired with elevated arms (pelvis to skull or skull base). Depending on the patient size and clinical indication, six to eight overlapping bed positions with 3 minutes of PET acquisition time each were used. Using the Biograph mCT 40 FLOW PET/CT scanner, images of the whole body (skull to feet) were acquired using the continuous bed move (torso: $0.8 \mathrm{~cm} / \mathrm{min}$, legs: $1.1 \mathrm{~cm} / \mathrm{min}$ ). The same area was covered by a low-dose 
CT scan (tube current $50 \mathrm{mAs}$, tube voltage $120 \mathrm{kV}$ ). No contrast agents were given.

PET images (slice thickness $5 \mathrm{~mm}$ ) were corrected for random coincidences, decay, scatter, and attenuation and reconstructed iteratively using the ordered subsets expectation maximization algorithm (OSEM) with four iterations and eight subsets. PET images were scaled to allow SUV measurements. PET and CT images were checked for breathing artifacts. Only PET images without ECG gating were used for re-analysis.

FDG PET/CT images were reanalyzed by two independent nuclear medicine physicians blinded to patients ' characteristics, using syngo.via software (version V30, Siemens Healthcare, Germany). Scans with abnormal focal or diffuse ${ }^{18}$ F-FDG uptake (without using a fixed threshold), compared to surrounding blood pool, corresponding to cardiac valve, prostheses or intracardiac devices were considered positive for IE. Attenuation corrected as well as uncorrected images were analyzed separately.

\section{Modified Duke classification}

DC were assessed at the time of admission, at the time of FDG PET/CT and at the end of hospital stay according to 2015 ESC IE guidelines. ${ }^{3}$

\section{Definition of final IE diagnosis}

Final IE diagnosis was made based on intraoperative findings being consistent with signs of acute or subacute infection (such as vegetations or abscesses). Microbiological tissue samples were documented positive when pathogens were successfully cultured or identified by PCR. Histopathological confirmation was gathered when histology was consistent with IE and/or pathogens could be identified.

\section{Statistical analysis}

Data were analyzed using the SPSS statistical software package (SPSS 23.0, IBM SPSS Statistics, Armonk, New York, USA). Descriptive statistics are presented as median and interquartile range (IQR) for continuous data and as number and percentages for categorical data. Fisher's exact test was used to compare median values for independent data. Categorical parameters were evaluated by Chi-squared test.

SEN was calculated with the following formula: number of true-positive test results/number of patients with surgically proven IE.

Due to selection of our patient cohort with high IE probability determination of specificity as well as positive and negative predictive value was not viable.

\section{RESULTS}

Between January 2014 and October 2018, 53 FDG PET/CT scans were performed in patients with both, ICD code for IE and OPS code for heart surgery. Out of 53 screened patients, $\mathrm{N}=21$ patients were excluded because FDG PET/CT scan was performed post-surgery. In two patients, intraoperative assessment could not confirm or reject IE diagnosis. In one patient, no FDG PET/CT images were available for re-analysis. Hence, 29 FDG PET/CT scans were eligible for retrospective analysis (Figure 1).

\section{Clinical characteristics}

Clinical characteristics of all 29 patients are displayed in Table 1. Median age was 64 years (IQR: 58 to 69 years) and the majority of patients was male (86\%). 8 patients $(28 \%)$ died during hospital stay. $69 \%$ of patients were referred from other hospitals. 7 patients $(24 \%)$ had native valve IE (NVE), 18 patients $(62 \%)$ had PVE and 4 patients $(14 \%)$ had isolated cardiac device-related IE (CDRIE). Vegetations were identified in 23 patients (79\%) by echocardiography and main IE affection site was aortic valve region. Median vegetation size was 17 mm (IQR: 12 to $23 \mathrm{~mm}$ ] and abscess formation was detected echocardiographically in 4 patients. $50 \%$ of PVE patients had bioprostheses and median time since prosthesis implantation was 4.0 years (IQR: .8 to 9.3 years). CRP at the time of admission was $148.7 \mathrm{mg} / \mathrm{dL}$ (IQR: 66.7 to $281.3 \mathrm{mg} / \mathrm{dL}$ ) and blood cultures were available in all patients. 26 patients $(90 \%)$ had positive blood cultures and the most frequent causative pathogen was Staphylococcus aureus (58\%). In 28 out of all 29 patients IE was proven surgically (1 patient had no in situ IE). Microbiology was available in 24 patients $(83 \%)$ and histopathology in 13 patients (45\%). FDG PET/CT was performed at a median time of 10 days (IQR: 7 to 20 days) in case of referral from another hospital and at a median time of 4 days (IQR: 3 to 8 days) after admission to our tertiary center. Time between first positive blood culture and FDG PET/CT was 8 days (IQR: 5 to 15 days). CRP at the time of FDG PET/CT was $87.3 \mathrm{mg} / \mathrm{dL}$ (IQR: 39.0-117.3 mg/dL) and 26 patients $(90 \%)$ received antibiotic therapy at the time of the scan.

\section{Test results of FDG PET/CT in patients with surgically managed IE}

Out of 29 FDG PET/CT scans only one was inconclusive. Hence, 28 FDG PET/CT scans results were used for further analysis. 15 patients with surgically confirmed IE had a positive FDG PET/CT 


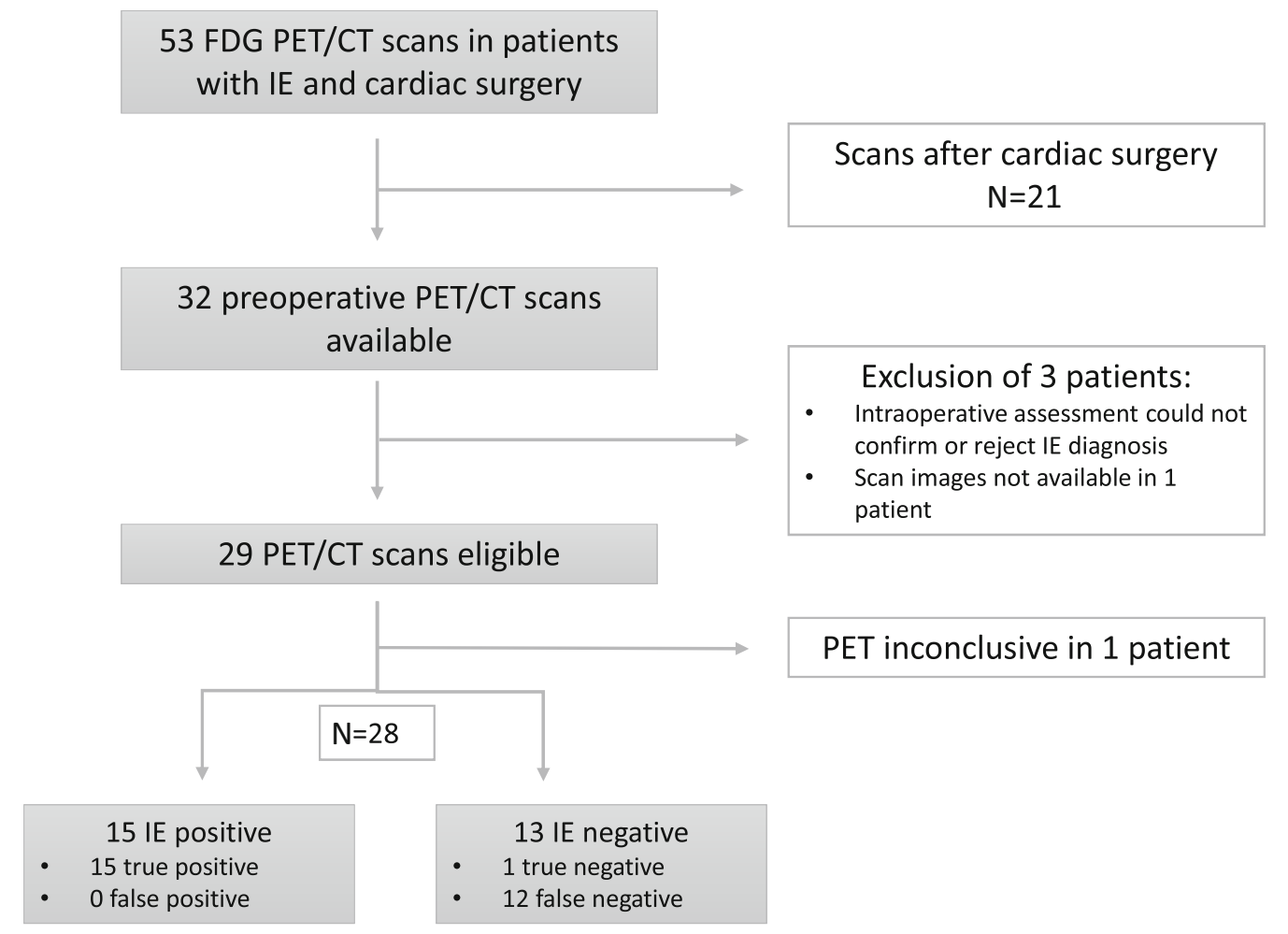

Figure 1. Flowchart of retrospective FDG PET/CT selection in patients with definite IE before cardiac surgery.

(sensitivity (SEN) 56\%). FDG PET/CT was negative in 13 patients, yet only 1 true-negative. Hence, 12 of 13 negative scan results were false-negative (Table 2). Exemplary FDG PET/CT scans and echocardiographic images illustrating the according endocarditic lesion are presented in Figure 2 for a patient with a true-positive test result (Figure 2A and B) and for a patient with a false-negative test result (Figure 2C and D).

Out of 15 patients with a positive FDG PET/CT scan, 12 patients had definite IE according to DC. In patients with a negative FDG PET/CT scan, diagnosis of IE according to DC would have been rejected in one patient with surgically confirmed IE. 22 out of 29 patients $(79 \%)$ fulfilled traditional DC for definite IE at the time of FDG PET/CT. Of these 22 patients, indications for FDG PET/CT were as follows: inconclusive results from echocardiography $(\mathrm{N}=4)$, other foci/septic emboli $(\mathrm{N}=7)$, combination of both $(\mathrm{N}=11)$. By including the FDG PET/CT result as a major DC into Duke classification, 3 further patients could be reclassified as definite IE (Figure 3). Combination of DC with FDG PET/CT scan resulted in a gain of SEN for all patients with confirmed IE (SEN of DC $79 \%$ vs SEN of combination DC and FDG PET/CT $89 \%$ ), driven by a relevant gain in PVE patients only
(SEN of DC 78\% vs SEN of combination DC and FDG PET/CT 94\%). In NVE, SEN of DC did not improve when combined with FDG PET/CT (SEN of DC $83 \%$ vs SEN of combination DC and FDG PET/CT 83\%). SEN values for DC, FDG PET/CT and combination of both are presented in Table 3 . Interestingly, 4 patients had septic emboli that were detected by FDG PET/CT. However, adding this finding as a minor DC did not result in a gain of IE SEN since all of the patients were diagnosed with definite IE according to DC at the time of FDG PET/CT already.

Table 4 depicts clinical characteristics of patients with true-positive and false-negative FDG PET/CT scans. Regarding demographic and echocardiographic parameters no significant differences between groups could be detected, yet a trend toward a greater proportion of female sex $(P=.053)$ and more echocardiographic evidence of abscess formation $(P=.053)$ could be seen in patients with true-positive scan results. Microbiological, histopathological and surgical parameters did not differ between groups. With respect to FDG PET/CT-specific parameters, true-positive scans showed as expected higher median SUVmax (5.8 [4.9-7.6] vs 3.9 [3.3-4.8], $P=.006$ ) compared to false-negative FDG PET/CTs. The proportion of patients 
Table 1. Clinical characteristics in patients with surgically managed IE and preoperatively performed FDG PET/CT

$\mathbf{N}=\mathbf{2 9}$

Demographics

Age, median [IQR], y

$64[58-69]$

Sex (male), $\mathrm{n}(\%)$

Diabetes mellitus, $\mathrm{n}(\%)$

$4(14)$

Prior history of IE, $\mathrm{n}(\%)$

$3(10)$

Intrahospital mortality, $\mathrm{n}(\%)$

$8(28)$

Intrahospital IE, $\mathrm{n}(\%)$

$3(10)$

Prior admission to other hospital, n (\%)

$20(69)$

Echocardiographic data

Native valve, $n$ (\%)

Impairment of LVEF, $\mathrm{n}(\%)$

Time to initial TOE, median [IQR], days

Time to TOE at tertiary hospital, median [IQR], days

Initial TOE-negative, $\mathrm{n}(\%)$

$7(24)$

$8(29)$

0 [-3 to 2$]$

$2.5[1-4]$

Primary Duke criterion-positive, $\mathrm{n}(\%)$

$1(4)$

Vegetation, $\mathrm{n}(\%)$

$27(93)$

$23(79)$

Vegetations only

$15 / 23$

IE affection site ${ }^{\mathrm{a}}, \mathrm{n}(\%)$

Aortic

$16(55)$

Mitral

$11(38)$

Pulmonary

$1(3)$

Tricuspid

$3(10)$

Cardiac device

7 (24)

Vegetation size, all, median [IQR], mm

17 [12-23]

NVE, median [IQR], mm

15 [12-25]

PVE, median [IQR], mm

16 [11-21]

Abscess, $\mathrm{n}(\%)$

$4(14)$

Fistula, n (\%)

$0(0)$

Prosthetic valve dehiscence, $\mathrm{n}(\%)$

$1(6)$

Paravalvular leakage, $\mathrm{n}(\%)$

$3(17)$

Prosthetic valve IE, $\mathrm{n}(\%)$

$18(62)$

Mechanical, $n$

$4 / 18$

Biological, $n$

$9 / 18$

$5 / 18$

Reconstruction, $\mathrm{n}$

$3 / 18$

Including replacement of ascending aorta, $\mathrm{n}$

$4.0[0.8-9.3]$

Time since implantation, median [IQR], y

$13 / 18$

Valves implanted $>1$ year, $\mathrm{n}$

$4 / 18$

Valves implanted $<3$ months, $\mathrm{n}$

$1 / 18$

Valves implanted 3-12 months, $n$

7 (24)

Cardiac device IE, $\mathrm{n}(\%)$

$4 / 7$

Isolated device infection, $\mathrm{n}$

$3 / 7$

Pacemaker, $\mathrm{n}$

$2 / 7$

$2 / 7$

CRT-D, $n$

$0 / 7$

CRT-P, $\mathrm{n}$

2 [1-4]

Time since implantation, median [IQR], y

$6 / 7$

Device implanted $>1$ year, $n$

$1 / 7$

Device implanted $<3$ months, $\mathrm{n}$ 
Table 1 continued

\begin{tabular}{|c|c|}
\hline & $\mathbf{N}=\mathbf{2 9}$ \\
\hline Device implanted 3-12 months, $\mathrm{n}$ & $0 / 7$ \\
\hline \multicolumn{2}{|l|}{ Microbiology } \\
\hline CRP at the time of admission, median [IQR], mg/L & 148.7 [66.7-281.3] \\
\hline Leukocytes at the time of admission, median [IQR], /nL & $11.8[8.9-15.3]$ \\
\hline PCT at the time of admission, median [IQR], $\mathrm{ng} / \mathrm{mL}$ & $1.33[1.02-19.52]$ \\
\hline Blood cultures available, $\mathrm{n}(\%)$ & $29(100)$ \\
\hline Blood cultures positive, $\mathrm{n}(\%)$ & $26(90)$ \\
\hline Primary Duke criterion positive, n (\%) & $22(76)$ \\
\hline \multicolumn{2}{|l|}{ Causative pathogen, $\mathrm{n}$} \\
\hline Staphylococcus aureus & $15 / 26$ \\
\hline Enterococci & $1 / 26$ \\
\hline Coagulase-negative staphylococci & $4 / 26$ \\
\hline Streptococci & $4 / 26$ \\
\hline HACEK & $1 / 26$ \\
\hline Candida sp. & $1 / 26$ \\
\hline Antibiotic therapy before blood cultures, $\mathrm{n}(\%)$ & $6(21)$ \\
\hline Empiric antibiotic therapy, $\mathrm{n}(\%)$ & $25(86)$ \\
\hline \multicolumn{2}{|l|}{ FDG PET/CT } \\
\hline Time to FDG PET/CT since external admission, median [IQR], days & $10[7-20]$ \\
\hline Time to FDG PET/CT since admission at tertiary center, median [IQR], days & $4[3-8]$ \\
\hline Time between FDG PET/CT and surgery, median [IQR], days & $9[4-16]$ \\
\hline \multicolumn{2}{|l|}{ Indication for FDG PET/CT } \\
\hline Inconclusive echocardiography & $5(17)$ \\
\hline Other foci/septic emboli & $11(38)$ \\
\hline Combination of both & $13(45)$ \\
\hline Time from first positive blood culture to FDG PET/CT, median [IQR], days & $8[5-15]$ \\
\hline Duration of antibiotic therapy before FDG PET/CT, median [IQR], days & $3[0-6]$ \\
\hline CRP at the time of FDG PET/CT, median [IQR], $\mathrm{mg} / \mathrm{L}$ & $87.3[39.0-117.3]$ \\
\hline Leukocytes at the time of FDG PET/CT, median [IQR], /nL & $10.7[7.5-12.8]$ \\
\hline Fasting glucose, median [IQR], mg/dL & $101[89-155]$ \\
\hline Fever at the time of FDG PET/CT, n (\%) & $3(18)$ \\
\hline FDG PET/CT during antibiotic therapy, $\mathrm{n}(\%)$ & $26(90)$ \\
\hline Pathogen-directed therapy, $\mathrm{n}(\%)$ & $21(72)$ \\
\hline Vegetation size at the time of FDG PET/CT, median [IQR], mm & $11[5-19]$ \\
\hline Inadequate myocardial suppression, $\mathrm{n}(\%)$ & $9(31)$ \\
\hline FDG PET/CT result inconclusive, $n(\%)$ & $1(3)$ \\
\hline FDG PET/CT positive, $\mathrm{n}$ & $15 / 28$ \\
\hline True-positive, $\mathrm{n}$ & $15 / 15$ \\
\hline False-positive, $\mathrm{n}$ & $0 / 15$ \\
\hline FDG PET/CT negative, $\mathrm{n}$ & $13 / 28$ \\
\hline True-negative, $\mathrm{n}$ & $1 / 13$ \\
\hline False-negative, $\mathrm{n}$ & $12 / 13$ \\
\hline SUVmax in positive FDG PET/CT, median [IQR] & $5.8[4.9-7.6]$ \\
\hline Septic emboli detected by FDG PET/CT, n (\%) & $4(14)$ \\
\hline Modified Duke classification at admission, n (\%) & $28(97)$ \\
\hline Definite IE, $n$ & $16 / 28$ \\
\hline Possible IE, $n$ & $7 / 28$ \\
\hline Rejected, $\mathrm{n}$ & $5 / 28$ \\
\hline Modified Duke classification at the time of FDG PET/CT, n (\%) & $29(100)$ \\
\hline Definite IE, $n$ & $22 / 29$ \\
\hline
\end{tabular}


Table 1 continued

\begin{tabular}{ll}
\hline & $\mathbf{N}=\mathbf{2 9}$ \\
\hline Possible IE, $\mathrm{n}$ & $6 / 29$ \\
Rejected, $\mathrm{n}$ & $1 / 29$ \\
Modified Duke classification at the end of hospital stay, $\mathrm{n}(\%)$ & $29(100)$ \\
Definite IE, $\mathrm{n}$ & $23 / 29$ \\
Possible IE, $\mathrm{n}$ & $6 / 29$ \\
Rejected, $\mathrm{n}$ & $0 / 29$ \\
Definite IE (microbiological, histopathological or surgical confirmation) & $24(83)$ \\
Microbiology available, $\mathrm{n}(\%)$ & $12 / 24$ \\
$\quad$ Microbiological confirmation of IE, $\mathrm{n}$ & $4 / 12$ \\
Culture positive, $\mathrm{n}$ & $11 / 12$ \\
PCR positive, $\mathrm{n}$ & $13(45)$ \\
Histopathology available, $\mathrm{n}(\%)$ & $10 / 13$ \\
$\quad$ Histopathological confirmation of IE, $\mathrm{n}$ & $6 / 10$ \\
$\quad$ Detection of germs, $\mathrm{n}$ & $28(97)$ \\
Surgical confirmation of IE, $\mathrm{n}(\%)$ & $11 / 28$
\end{tabular}

$C R P$, C-reactive protein; $C R T-D$, cardiac resynchronization therapy defibrillator; $C R T-P$, cardiac resynchronization therapy pacemaker; $C T$, computed tomography; $F D G,{ }^{18} \mathrm{~F}$-fluorodeoxyglucose; $I C D$, implantable cardioverter defibrillator; $I E$, infective endocarditis; $L V E F$, left ventricular ejection fraction; $P C T$, procalcitonin; $P E T$, positron emission tomography; SUVmax, maximal standardized uptake value; TOE, transesophageal echocardiography

Values represent the median [interquartile range] or numbers (percentages)

${ }^{a}$ The number of IE affection sites is higher than the number of patients because some patients had more than one affection site

Table 2. Two-by-two contingency table for the diagnosis of infective endocarditis via FDG PET/ CT

\begin{tabular}{lccc}
\hline & \multicolumn{3}{c}{ Reference standard $^{\mathbf{a}}$} \\
\cline { 2 - 4 } & + & - & Total \\
\hline PET & & & \\
+ & 15 & 0 & 15 \\
- & 12 & 1 & 13 \\
Total & 27 & 1 & 28 \\
\hline
\end{tabular}

PET, positron emission tomography

${ }^{a}$ Reference standard corresponds to intraoperative confirmation of infective endocarditis

with inadequate myocardial suppression was not significantly different between both groups.

In case of PVE type of prosthesis was not different between groups, however both, time since prosthesis implantation (1.0 year vs 6.5 years, $P=.035)$ and proportion of patients with prosthesis implanted longer than one year ago $(55 \%$ vs $100 \%, P=.049)$, were greater in patients with false-negative FDG PET/CT scans.

\section{DISCUSSION}

In our retrospective analysis of surgically managed IE cases diagnosis of definite IE was confirmed in 28 of 29 patients via intraoperative assessment. This way of definite IE diagnosis is a major strength of our study, because final IE diagnosis was not based on DC or expert opinion only, but was surgically proven, therefore correlation with PET imaging is reliable. SEN of DC at the time of PET imaging was $79 \%$ overall and $78 \%$ for PVE, SEN of preoperatively performed FDG PET/CTs was $56 \%$ overall and $61 \%$ in PVE. Including positive FDG PET/CT scans as a major DC resulted in a gain of modified DC SEN in PVE cases by $16 \%$ (3 out of 4 patients with possible PVE were reclassified to definite PVE).

SEN values of DC range between 70 and $80 \%$ in literature, ${ }^{11}$ for PVE due to challenges in echocardiographic image acquisition and interpretation even lower values are observed. ${ }^{10}$ Hence, DC SEN values as detected in our study are well within the range of reported DC SEN.

In contrast, FDG PET/CT SEN in our current study was notably lower than SEN values in other studies. A meta-analysis of 13 studies involving 537 patients as published in 2017 found a pooled SEN of FDG PET/CT 
A

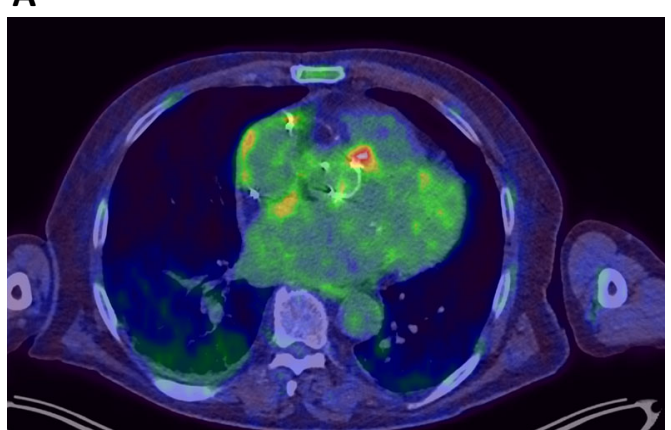

C

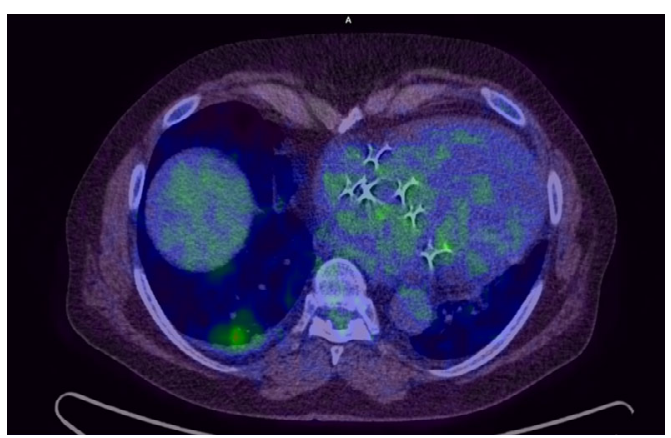

B

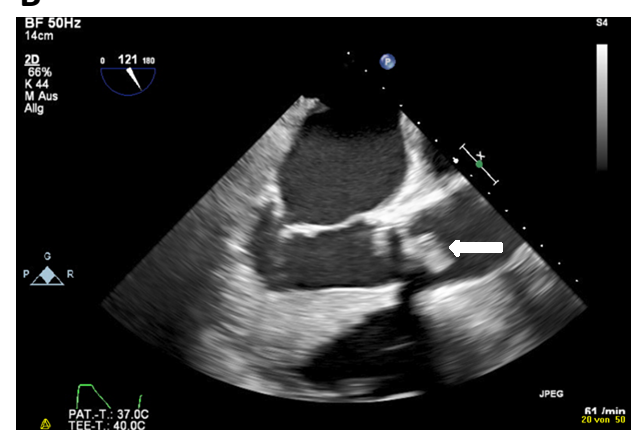

D

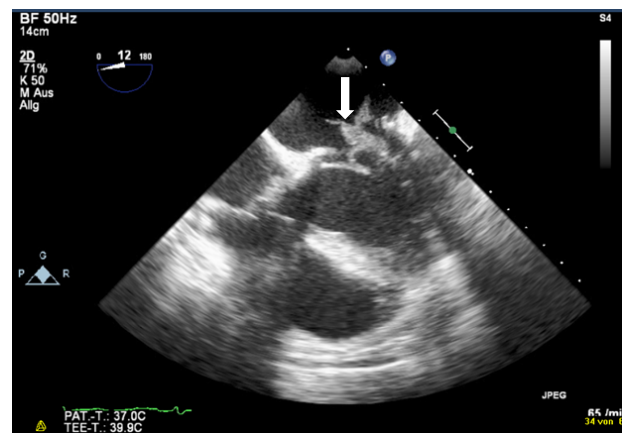

Figure 2. Exemplary FDG PET/CT scans and echocardiographic images illustrating the according endocarditic lesion are presented in this figure for a patient with a true-positive test result (A and $\mathbf{B})$ and for a patient with a false-negative test result $(\mathbf{C}$ and $\mathbf{D})$.

A

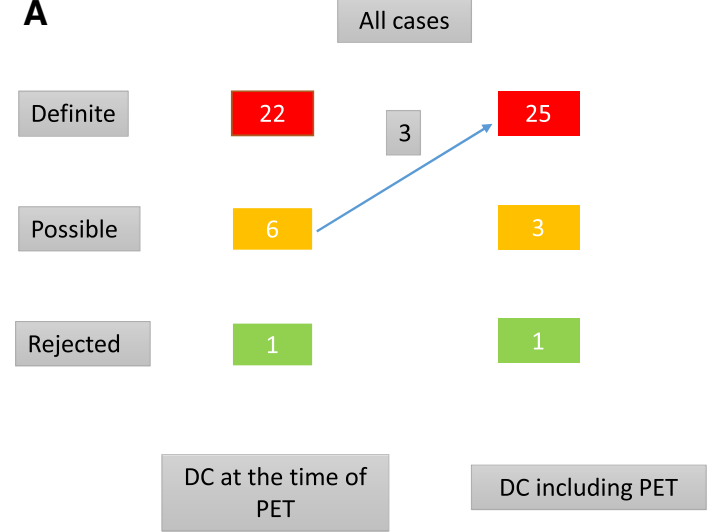

B

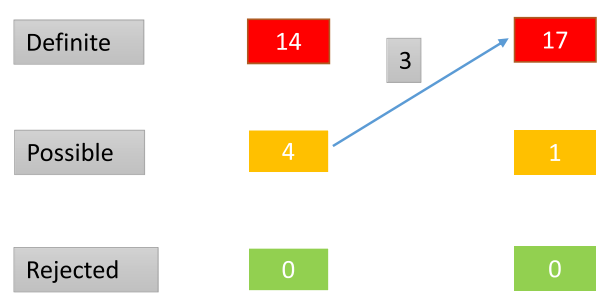

Figure 3. Reclassification of subjects with possible IE according to DC at the time of PET using positive FDG PET/CT result as major DC (A: all cases, B: PVE).

of $76.8 \%$ for all IE, respectively, of $80.5 \%$ for PVE. ${ }^{12}$ This discrepancy is somewhat surprising, especially since patients in our current study were all treated surgically suggesting a rather advanced stage of IE that should be more prone to pathologic FDG/PET CT scans. However, it needs to be mentioned that patients in our retrospective analysis represent an unselected real-world cohort of complexly diseased patients that ultimately required surgical therapy due to IE. Hence, indication for FDG PET/CT was not based on current ESC guidelines only. In fact, patients were mostly referred to the Department of Nuclear Medicine for the detection 
Table 3. Sensitivity values of DC, FDG PET/CT, and combination of DC and FDG PET/CT for all patients as well as in NVE, PVE and CDRIE

\begin{tabular}{lccc}
\hline & SEN DC & SEN PET/CT & SEN DC + PET/CT \\
\hline All $(\mathrm{N}=28)$ & $79 \%(60-90 \%)$ & $56 \%(37-72 \%)$ & $89 \%(73-96 \%)$ \\
NVE $(\mathrm{N}=6)$ & $83 \%(44-97 \%)$ & $33 \%(10-70 \%)$ & $83 \%(44-97 \%)$ \\
PVE $(\mathrm{N}=18)$ & $78 \%(55-91 \%)$ & $61 \%(39-80 \%)$ & $94 \%(74-99 \%)$ \\
CDRIE $(\mathrm{N}=4)$ & $75 \%(30-95 \%)$ & $50 \%(15-85 \%)$ & $75 \%(30-95 \%)$
\end{tabular}

$C D R I E$, cardiac device-related infective endocarditis; $C T$, computed tomography; $D C$, Duke criteria; $F D G,{ }^{18} \mathrm{~F}$-fluorodeoxyglucose; $N V E$, native valve endocarditis; $P E T$, positron emission tomography; PVE, prosthetic valve endocarditis; SEN, sensitivity

Values represent percentages ( $95 \%$ confidence interval)

${ }^{\mathrm{a} D C}$ at the time of PET/CT

of an infectious focus in general. This is why usually recommended technical requirements such as the suppression of myocardial nuclide uptake or ECG-gated PET image acquisition were not routinely performed. These limitations in patient preparation and image acquisition may have likely contributed to the substantially lower sensitivity of FDG PET/CT in our study population that, however, still resulted in a relevant gain of diagnosing IE when using modified DC.

When comparing patients with true-positive vs false-negative scans in our study, median time since prosthesis valve implantation was significantly longer in the group with false-negative scans (median prosthesis age 6.5 years). In addition, proportion of patients with prostheses implanted longer than 1 year ago was significantly higher in patients with false-negative scans. Considering the lower PET/CT SEN in NVE ${ }^{13,14}$ this finding may be hypothesis generating in a way that valve prostheses may align their FDG PET/CT enhancement profile to NVE levels over time resulting in more falsenegative scans. To the best of our knowledge this potential correlation has not been described before and should be taken into consideration when interpreting FDG PET/CT scans in patients with older valve prostheses.

Furthermore, we observed a trend toward less echocardiographically detected abscess formation $(P=.053)$ and less female sex $(P=.053)$ in patients with false-negative scans, yet without statistical significance. The former may suggest less inflammatory activity in patients with false-negative FDG PET/CT scans. However, in our study neither CRP nor leukocyte levels differed between both groups. This is in contrast to a study by Swart et al in which potential confounders of false-negative PET/CT scans in PVE patients were investigated and low inflammatory activity, namely low CRP levels at the time of PET imaging was described as a confounding factor resulting in false-negative scans. ${ }^{10}$
This discrepancy is hard to explain, but may be due to the fact that Swart $e t$ al have analyzed patients with less elevated overall inflammatory activity (CRP around 50$60 \mathrm{mg} / \mathrm{L}$ ) who required surgical therapy in only half of the cases. Of note, patients in our study had higher CRP levels (ranging from 81-106 mg/L) and were all treated surgically, yet had a high proportion of false-negative FDG PET/CT scans. This may suggest a threshold from which elevated CRP levels in IE patients rather reflect the systemic inflammatory response as compared to the localized inflammatory activity within the IE affection site, and high CRP levels do not necessarily exclude the possibility of false-negative results.

Up to now, only one other study has investigated patients with preoperatively performed FDG PET/CTs and surgically managed IE. ${ }^{15}$ El-Dalati et al reported 12 true-positive FDG PET/CT scans in 12 patients with surgically proven IE (i.e., a SEN of 100\%). Possible influencing factors like proportion of PVE, distribution of causative pathogens or surgical findings do not suggest such contradictory data. While a comprehensive explanation of these conflicting findings may be still limited to small sample size of both studies, further details of studied patients (e.g., time since prosthesis valve implantation or gender distribution) would be of interest in order to better understand possible confounders.

We only found few cases with NVE and cardiac device-related IE (CDRIE) in our study. In that scenario sensitivity of PET imaging was low (SEN NVE 33\%, SEN CDRIE 50\%) and addition to traditional DC did not result in a gain of SEN. For NVE low PET SEN has been reported so far with values ranging from $22 \%$ to $45 \%,{ }^{13,14}$ in contrast Abikhzer et al. reported on notably higher SEN values 68\%. ${ }^{9}$ Data to SEN in case of CDRIE are ranged from very low (16.3\% in the ESC-EORPEURO-ENDO study ${ }^{14}$ ) up to high values of $>85 \%$. 
Table 4. Clinical characteristics in IE patients with true-positive vs false-negative FDG PET/CT results

\begin{tabular}{|c|c|c|c|}
\hline & $\begin{array}{c}\text { FDG PET/CT true- } \\
\text { positive } \\
(\mathbf{N}=15)\end{array}$ & $\begin{array}{l}\text { FDG PET/CT false- } \\
\text { negative } \\
(N=12)\end{array}$ & $\underset{\text { value }}{P}$ \\
\hline \multicolumn{4}{|l|}{ Demographics } \\
\hline Age, median [IQR], y & $66[53-75]$ & $64[62-67]$ & .441 \\
\hline Sex (male), n (\%) & $11(73)$ & $12(100)$ & .053 \\
\hline Diabetes mellitus, n (\%) & $3(20)$ & $0(0)$ & .1 \\
\hline Prior history of IE, n (\%) & $1(7)$ & $1(8)$ & .509 \\
\hline Intrahospital mortality, n (\%) & $5(33)$ & $3(25)$ & .637 \\
\hline Intrahospital IE, n (\%) & $1(7)$ & $2(17)$ & .411 \\
\hline Prior admission to other hospital, $\mathrm{n}(\%)$ & $12(80)$ & $12(80)$ & .432 \\
\hline \multicolumn{4}{|l|}{ Echocardiographic data } \\
\hline Native valve, n (\%) & $2(15)$ & $4(40)$ & 198 \\
\hline Impairment of LVEF, n (\%) & $4(29)$ & $4(33)$ & .793 \\
\hline Time to initial TOE, median [IQR], days & $0[-4-2]$ & $1[-2-5]$ & .685 \\
\hline Time to TOE at tertiary hospital, median [IQR], days & $3[1-3.5]$ & $4[1-6]$ & 187 \\
\hline Initial TOE-negative, n (\%) & $1(7)$ & $0(0)$ & .327 \\
\hline Primary Duke criterion-positive, n (\%) & $14(93)$ & $11(92)$ & .358 \\
\hline Vegetation, n (\%) & $12(80)$ & $10(83)$ & .651 \\
\hline Vegetations only & $8 / 12$ & $7 / 10$ & .951 \\
\hline \multicolumn{4}{|l|}{ IE affection site ${ }^{\mathrm{a}}$} \\
\hline Aortic & $9(60)$ & $6(50)$ & .603 \\
\hline Mitral & $4(27)$ & $6(50)$ & .212 \\
\hline Pulmonary & $0(0)$ & $1(8)$ & .255 \\
\hline Tricuspid & $3(20)$ & $0(0)$ & .1 \\
\hline Cardiac device & $3(20)$ & $4(33)$ & .432 \\
\hline Vegetation size, median [IQR], mm & 19 [14-23] & $17[11-22]$ & 1 \\
\hline Vegetation size in NVE, median, mm & $20^{\mathrm{b}}$ & $18^{\mathrm{b}}$ & 1.0 \\
\hline Abscess, n (\%) & $4(27)$ & $0(0)$ & .053 \\
\hline Fistula, n (\%) & $0(0)$ & $0(0)$ & 1 \\
\hline Prosthetic valve dehiscence, n (\%) & $0(0)$ & $1(17)$ & .171 \\
\hline Paravalvular leakage, n (\%) & $2(18)$ & $1(17)$ & .829 \\
\hline Prosthetic valve IE, n (\%) & $11(73)$ & $6(50)$ & .212 \\
\hline Mechanical, n & $2 / 11$ & $2 / 6$ & .482 \\
\hline Biological, n & $6 / 11$ & $3 / 6$ & .858 \\
\hline Reconstruction, $\mathrm{n}$ & $3 / 11$ & $1 / 6$ & .622 \\
\hline Including replacement of ascending aorta, $n$ & $2 / 11$ & $0 / 6$ & .266 \\
\hline Vegetation size, median [IQR], mm & $16[12-21]$ & $14[11-21]$ & .825 \\
\hline Time since implantation, median [IQR], y & $1.0[.0-4.0]$ & $6.5[4.8-14.8]$ & .035 \\
\hline Valves implanted $>1$ year, $\mathrm{n}$ & $6 / 11$ & $6 / 6$ & .049 \\
\hline Valves implanted $<3$ months, $\mathrm{n}$ & $4 / 11$ & $0 / 6$ & .091 \\
\hline Valves implanted 3-12 months, $\mathrm{n}$ & $1 / 11$ & $0 / 6$ & .446 \\
\hline Cardiac device IE, n (\%) & $3(10)$ & $4(33)$ & .432 \\
\hline Isolated device infection, $\mathrm{n}$ & $2 / 3$ & $2 / 4$ & .659 \\
\hline Pacemaker, n & $2 / 3$ & $1 / 4$ & .27 \\
\hline ICD, $\mathrm{n}$ & $1 / 3$ & $1 / 4$ & .809 \\
\hline CRT-D, n & $0 / 3$ & $2 / 4$ & .147 \\
\hline CRT-P, n & $0 / 3$ & $0 / 4$ & 1 \\
\hline Time since implantation, median [IQR], y & $2.0^{\mathrm{b}}$ & $2.5[1.3-3.8]$ & 1 \\
\hline Device implanted $>1$ year, $\mathrm{n}$ & $2 / 3$ & $4 / 4$ & .212 \\
\hline Device implanted $<3$ months, $\mathrm{n}$ & $1 / 3$ & $0 / 4$ & .212 \\
\hline
\end{tabular}


Table 4 continued

\begin{tabular}{|c|c|c|c|}
\hline & $\begin{array}{l}\text { FDG PET/CT true- } \\
\text { positive } \\
(\mathbf{N}=15)\end{array}$ & $\begin{array}{l}\text { FDG PET/CT false- } \\
\text { negative } \\
(\mathrm{N}=12)\end{array}$ & $\begin{array}{l}P \\
\text { value }\end{array}$ \\
\hline Device implanted 3-12 months, $\mathrm{n}$ & $0 / 3$ & $0 / 4$ & 1 \\
\hline \multicolumn{4}{|l|}{ Microbiology } \\
\hline CRP at the time of admission, median [IQR], $\mathrm{mg} / \mathrm{L}$ & $168.1[109.0-278]$ & $103.5[41.4-343.8]$ & .637 \\
\hline $\begin{array}{l}\text { Leukocytes at the time of admission, median [IQR], / } \\
\mathrm{nL}\end{array}$ & $12.6[9.6-19.1]$ & $11.1[6.4-14.7]$ & .704 \\
\hline $\mathrm{PCT}$ at the time of admission, median [IQR], $\mathrm{ng} / \mathrm{mL}$ & $1.37[1.09-23.51]$ & $9.20[0.86-21.38]$ & 1 \\
\hline Blood cultures available, n (\%) & $15(100)$ & $12(100)$ & 1 \\
\hline Blood cultures-positive, n (\%) & $15(100)$ & $10(83)$ & .1 \\
\hline Primary Duke criterion-positive, n (\%) & $13(87)$ & $9(75)$ & .438 \\
\hline \multicolumn{4}{|l|}{ Causative pathogen } \\
\hline Staphylococcus aureus & $9 / 15$ & $6 / 10$ & 1 \\
\hline Enterococci & $0 / 15$ & $1 / 10$ & .211 \\
\hline Coagulase-negative staphylococci & $3 / 15$ & $1 / 10$ & .504 \\
\hline Streptococci & $1 / 15$ & $2 / 10$ & .315 \\
\hline HACEK & $1 / 15$ & $0 / 10$ & .405 \\
\hline Candida sp. & $1 / 15$ & $0 / 10$ & .405 \\
\hline Antibiotic therapy before blood cultures, n (\%) & $3(20)$ & $2(17)$ & .923 \\
\hline Empiric antibiotic therapy, $\mathrm{n}(\%)$ & $14(93)$ & $10(83)$ & .283 \\
\hline \multicolumn{4}{|l|}{ FDG PET/CT } \\
\hline $\begin{array}{l}\text { Time to FDG PET/CT since external admission, } \\
\text { median [IQR], days }\end{array}$ & $10[5-19]$ & $11[7-30]$ & 1 \\
\hline $\begin{array}{l}\text { Time to FDG PET/CT since admission at tertiary } \\
\text { center, median [IQR], days }\end{array}$ & $4[3-7]$ & $5[3-8]$ & .449 \\
\hline $\begin{array}{l}\text { Time between FDG PET/CT and surgery, median } \\
{[I Q R] \text {, days }}\end{array}$ & $11[5-20]$ & $6[4-12]$ & .126 \\
\hline $\begin{array}{l}\text { Time from first-positive blood culture to FDG PET/CT, } \\
\text { median [IQR], days }\end{array}$ & $8[4-17]$ & $8[7-15]$ & .669 \\
\hline $\begin{array}{l}\text { Duration of antibiotic therapy before FDG PET/CT, } \\
\text { median [IQR], days }\end{array}$ & $1[0-11]$ & $3[-6-5]$ & .605 \\
\hline CRP at the time of FDG PET/CT, median [IQR], $\mathrm{mg} / \mathrm{L}$ & $81.2[34.4-102.5]$ & $106.0[71.2-193.5]$ & .68 \\
\hline $\begin{array}{l}\text { Leukocytes at the time of FDG PET/CT, median [IQR], } \\
/ \mathrm{nL}\end{array}$ & $10.8[9.2-12.9]$ & $9.4[6.4-12.9]$ & 1 \\
\hline Fasting glucose, median [IQR], mg/dL & $95[88-138]$ & $103[93-144]$ & .697 \\
\hline Fever at the time of FDG PET/CT, $n(\%)$ & $1(7)$ & $2(17)$ & .522 \\
\hline FDG PET/CT during antibiotic therapy, n (\%) & $14(93)$ & $10(83)$ & .185 \\
\hline Pathogen-directed therapy, n (\%) & $11(73)$ & $8(67)$ & .702 \\
\hline $\begin{array}{l}\text { Vegetation size at the time of FDG PET/CT, median } \\
\text { [IQR], mm }\end{array}$ & $10[0-16]$ & $17[9-21]$ & .4 \\
\hline Inadequate myocardial suppression, n (\%) & $4(27)$ & $4(33)$ & .706 \\
\hline SUVmax in positive FDG PET/CT, median [IQR] & $5.8[4.9-7.6]$ & $3.9[3.3-4.8]$ & .006 \\
\hline Septic emboli detected by FDG PET/CT, n (\%) & $2(13)$ & $2(17)$ & .809 \\
\hline Modified Duke classification at admission, $\mathrm{n}(\%)$ & $14(93)$ & $12(100)$ & .362 \\
\hline Definite IE, $\mathrm{n}$ & $9 / 14$ & $6 / 12$ & .462 \\
\hline Possible IE, $n$ & $4 / 14$ & $3 / 12$ & .838 \\
\hline Rejected, $\mathrm{n}$ & $1 / 14$ & $3 / 12$ & .208 \\
\hline $\begin{array}{l}\text { Modified Duke classification at time of FDG PET/CT, } n \\
(\%)\end{array}$ & $15(100)$ & $12(100)$ & 1 \\
\hline Definite IE, $n$ & $12 / 15$ & $9 / 12$ & .756 \\
\hline
\end{tabular}


Table 4 continued

\begin{tabular}{|c|c|c|c|}
\hline & $\begin{array}{l}\text { FDG PET/CT true- } \\
\text { positive } \\
(\mathbf{N}=15)\end{array}$ & $\begin{array}{l}\text { FDG PET/CT false- } \\
\text { negative } \\
(\mathrm{N}=12)\end{array}$ & $\begin{array}{l}P \\
\text { value }\end{array}$ \\
\hline Possible IE, $n$ & $3 / 15$ & $2 / 12$ & .825 \\
\hline Rejected, $\mathrm{n}$ & $0 / 15$ & $1 / 12$ & .255 \\
\hline $\begin{array}{l}\text { Modified Duke classification at end of hospital stay, } n \\
(\%)\end{array}$ & $15(100)$ & $12(100)$ & 1 \\
\hline Definite IE, $\mathrm{n}$ & $14 / 15$ & $8 / 12$ & .076 \\
\hline Possible IE, $n$ & $1 / 15$ & $4 / 12$ & .076 \\
\hline Rejected, $\mathrm{n}$ & $0 / 15$ & $0 / 12$ & 1 \\
\hline \multicolumn{4}{|l|}{$\begin{array}{l}\text { Definite IE (microbiological, histopathological or } \\
\text { surgical confirmation) }\end{array}$} \\
\hline Microbiology available, n (\%) & $14(93)$ & $10(83)$ & .411 \\
\hline Microbiological confirmation of IE, $\mathrm{n}$ & $7 / 14$ & $5 / 10$ & 1 \\
\hline Culture-positive, $n$ & $1 / 7$ & $3 / 5$ & .098 \\
\hline PCR-positive, $\mathrm{n}$ & $7 / 7$ & $4 / 5$ & .217 \\
\hline Histopathology available, n (\%) & $8(53)$ & $4(33)$ & 299 \\
\hline Histopathological confirmation of IE, $n$ & $7 / 8$ & $2 / 4$ & .253 \\
\hline Detection of germs, $\mathrm{n}$ & $4 / 7$ & $1 / 2$ & .858 \\
\hline Surgical confirmation of IE, n (\%) & $15(100)$ & $12(100)$ & 1 \\
\hline Intraoperative abscess, n & $7 / 15$ & $3 / 12$ & .247 \\
\hline
\end{tabular}

CRP, C-reactive protein; CRT-D, cardiac resynchronization therapy defibrillator; CRT-P, cardiac resynchronization therapy pacemaker; CT, computed tomography; FDG, ${ }^{18} \mathrm{~F}$-fluorodeoxyglucose; ICD, implantable cardioverter defibrillator; IE, infective endocarditis; LVEF, left ventricular ejection fraction; PCT, procalcitonin; PET, positron emission tomography; SUVmax, maximal standardized uptake value; TOE, transesophageal echocardiography

Values represent the median [interquartile range] or numbers (percentages)

${ }^{a}$ The number of IE affection sites is higher than the number of patients because some patients had more than one affection site

b Interquartile range could not be calculated as $n<4$

These conflicting data probably represent the heterogenic study situation in this highly complex disease.

According to the current ESC IE guidelines PET imaging should only be performed in suspected PVE with prostheses implanted more than 3 months ago to avoid false-positive scans as a result of postoperative inflammation. We included 4 patients with prostheses implanted less than 3 months ago and all of them had right positive FDG PET/CT scans. Despite lack of statistical significance this observation corroborates results from other studies ${ }^{10,16}$ questioning this 3 months safety period.

\section{LIMITATIONS}

An important limitation of our study is the small number of patients in each group and the resulting lack of statistical power. Furthermore, due to the retrospective study design indication for FDG PET/CT was not based on current ESC guidelines only, but included also patients with definite IE diagnosis according to traditional DC which could lead to an important selection bias in this study.

Furthermore, our study may be influenced by a selection bias from identifying patients by the diagnosis and diagnostic procedures coded at the end of hospitalization for the statutory health insurance. From this reason, we expect a high prevalence of IE in our cohort. Because of our highly selected patient cohort determination of FDG PET/CT specificity as well as positive and negative predictive value is not viable. Unfortunately, data on pathological and microbiological evaluation of tissue samples were not available in all cases, which is relevant since definition of definite IE according to the current ESC IE guidelines does not involve sole confirmation of IE by the surgeon. ECG gating of PET images may increase detectability of small foci with elevated FDG uptake. In this retrospective study, only ungated PET images were analyzed as not all FDG PET/CTs were acquired with ECG gating, 
because most patients were referred to whole body PET/ CT and not to dedicated cardiac PET imaging. Similarly, suppression of the myocardial nuclide uptake was not routinely performed, since the patients were referred to the Department of Nuclear Medicine for the detection of an infectious focus in general. These limitations in patient preparation and image acquisition may have likely contributed to the substantially lower sensitivity of FDG PET/CT in our study population.

\section{CONCLUSION}

Our findings support the recommendation of current ESC IE guidelines for use of FDG PET/CT as complementary imaging to increase SEN of modified DC in PVE. However, we found evidence that increasing prosthesis age corresponds with lower SEN and therefore negative PET imaging should be interpreted with caution. Prospective trials are needed to better understand the value of FDG PET/CT in diagnosing IE.

\section{NEW KNOWLEDGE GAINED}

Our retrospective analysis that uses surgical assessment of definite IE diagnosis as reference standard gains knowledge with respect to the SEN of FDG PET/CT scans in a real-word cohort of unselected patients with surgically managed IE. We found a relevant gain of modified DC SEN (i.e., including FDG PET/CT scans and DC) in PVE patients only (3 out of 4 possible PVE patients could be reclassified to definite IE). PVE patients with false-negative FDG PET/CT results had significantly older valve prostheses, which should be considered when interpreting FDG PET/CT scans of IE patients.

\section{Author contribution}

All authors contributed to the study conception and design. Material preparation, data collection and analysis were performed by Sabine Julia Maria Sag, Can Martin Sag, Jirka Grosse and Karin Menhart. The first draft of the manuscript was written by Sabine Julia Maria Sag and all authors commented on previous versions of the manuscript. All authors read and approved the final manuscript.

\section{Funding} $D E A L$.

Open Access funding enabled and organized by Projekt

\section{Disclosures}

Sabine Julia Maria Sag, Karin Menhart, Jirka Grosse, Dirk Hellwig, Florian Hitzenbichler, Frank Hanses, Arno Mohr, Bernd Salzberger, Matthäus Zerdzitzki, Michael Hilker,
Leopold Rupprecht, Christof Schmid, Lars Siegfried Maier and Can Martin Sag declare that they have no conflict of interest.

\section{Ethical approval}

This retrospective analysis of existing patient data and images was approved by the local ethics committee (No. 18972-104).

\section{Informed consent}

Requirement for written informed consent was waived for our retrospective study.

\section{Open Access}

This article is licensed under a Creative Commons Attribution 4.0 International License, which permits use, sharing, adaptation, distribution and reproduction in any medium or format, as long as you give appropriate credit to the original author(s) and the source, provide a link to the Creative Commons licence, and indicate if changes were made. The images or other third party material in this article are included in the article's Creative Commons licence, unless indicated otherwise in a credit line to the material. If material is not included in the article's Creative Commons licence and your intended use is not permitted by statutory regulation or exceeds the permitted use, you will need to obtain permission directly from the copyright holder. To view a copy of this licence, visit http://creativecommons.org/licenses/by/4.0/.

\section{References}

1. Habib G. Management of infective endocarditis. Heart. 2006;92:124-30.

2. Habib G, Badano L, Tribouilloy C, Vilacosta I, Zamorano JL, Galderisi $\mathrm{M}$, et al. Recommendations for the practice of echocardiography in infective endocarditis. Eur $\mathbf{J}$ Echocardiogr. 2010;11:202-19.

3. Habib G, Lancellotti P, Antunes MJ, Bongiorni MG, Casalta J-P, Del Zotti F, et al. 2015 ESC Guidelines for the management of infective endocarditis: The Task Force for the Management of Infective Endocarditis of the European Society of Cardiology (ESC)Endorsed by: European Association for Cardio-Thoracic Surgery (EACTS), the European Association of Nuclear Medicine (EANM). Eur Heart J. 2015;36:3075-128.

4. Saby L, Laas O, Habib G, Cammilleri S, Mancini J, Tessonnier L, et al. Positron Emission Tomography/Computed Tomography for Diagnosis of Prosthetic Valve Endocarditis: Increased Valvular 18F-Fluorodeoxyglucose Uptake as a Novel Major Criterion. Journal of the American College of Cardiology. 2013;61:2374-82.

5. Sarrazin J-F, Philippon F, Tessier M, Guimond J, Molin F, Champagne J, et al. Usefulness of Fluorine-18 Positron Emission Tomography/Computed Tomography for Identification of Cardiovascular Implantable Electronic Device Infections. Journal of the American College of Cardiology. 2012;59:1616-25.

6. Bensimhon L, Lavergne T, Hugonnet F, Mainardi JL, Latremouille C, Maunoury $\mathrm{C}$, et al. Whole body [18F]fluorodeoxyglucose positron emission tomography imaging for the diagnosis of pacemaker or implantable cardioverter 
defibrillator infection: a preliminary prospective study. Clinical Microbiology and Infection. 2011;17:836-44.

7. Pizzi MN, Roque A, Fernández-Hidalgo N, Cuéllar-Calabria H, Ferreira-González I, Gonzàlez-Alujas MT, et al. Improving the Diagnosis of Infective Endocarditis in Prosthetic Valves and Intracardiac Devices With 18F-Fluordeoxyglucose Positron Emission Tomography/Computed Tomography Angiography. Circulation. 2015;132:1113-26.

8. Sánchez-Enrique C, Olmos C, Jiménez-Ballvé A, Fernández-Pérez C, Ferrera C, Pérez-Castejón MJ, et al. Usefulness of 18F Fluorodeoxyglucose Positron Emission Tomography/Computed Tomography in Infective Endocarditis in Daily Practice: Individualized Analysis of Each Potential Focus of Infection: A Prospective Cohort Study. JACC: Cardiovascular Imaging 2018;11:1920-2.

9. Abikhzer G, Martineau P, Grégoire J, Finnerty V, Harel F, Pelletier-Galarneau M. [18F]FDG-PET CT for the evaluation of native valve endocarditis. Journal of Nuclear Cardiology 2020.

10. Swart LE, Gomes A, Scholtens AM, Sinha B, Tanis W, Lam $\mathrm{MGEH}$, et al. Improving the Diagnostic Performance of $18 \mathrm{~F}$ Fluorodeoxyglucose Positron-Emission Tomography/Computed Tomography in Prosthetic Heart Valve Endocarditis. Circulation. 2018;138:1412-27.

11. Habib G, Derumeaux G, Avierinos J-F, Casalta J-P, Jamal F, Volot $\mathrm{F}$, et al. Value and limitations of the duke criteria for the diagnosis of infective endocarditis. Journal of the American College of Cardiology 1999;33:2023-9. (http://www.sciencedirect.com/scien ce/article/pii/S0735109799001163).
12. Mahmood M, Kendi AT, Ajmal S, Farid S, O'Horo JC, Chareonthaitawee $\mathrm{P}$, et al. Meta-analysis of 18F-FDG PET/CT in the diagnosis of infective endocarditis. Journal of Nuclear Cardiology. 2019;26:922-35.

13. Kouijzer IJ, Berrevoets MA, Aarntzen EH, de Vries J, van Dijk $\mathrm{AP}$, Oyen WJ, et al. 18F-fluorodeoxyglucose positron-emission tomography combined with computed tomography as a diagnostic tool in native valve endocarditis. Nuclear Medicine Communications. 2018;39:747-52.

14. Habib G, Erba PA, Iung B, Donal E, Cosyns B, Laroche C, et al. Clinical presentation, aetiology and outcome of infective endocarditis. Results of the ESC-EORP EURO-ENDO (European infective endocarditis) registry: a prospective cohort study. Eur Heart J 2019;40:3222-32.

15. El-Dalati S, Murthy VL, Owczarczyk AB, Fagan C, Riddell J, Cinti S, et al. Correlating cardiac F-18 FDG PET/CT results with intra-operative findings in infectious endocarditis. Journal of Nuclear Cardiology 2019.

16. Roque A, Pizzi MN, Fernández-Hidalgo N, Permanyer E, CuellarCalabria H, Romero-Farina G, et al. Morpho-metabolic post-surgical patterns of non-infected prosthetic heart valves by [18F]FDG PET/CTA: "normality" is a possible diagnosis. Eur Heart J Cardiovasc Imaging. 2019;21:24-33.

Publisher's Note Springer Nature remains neutral with regard to jurisdictional claims in published maps and institutional affiliations. 